\title{
BMJ Open Assessing the effect of an interactive decision-aid smartphone smoking cessation application (app) on quit rates: a double-blind automated randomised control trial protocol
}

\author{
Nasser F BinDhim, ${ }^{1,2}$ Kevin McGeechan, ${ }^{1}$ Lyndal Trevena ${ }^{1}$
}

To cite: BinDhim NF, McGeechan K, Trevena L. Assessing the effect of an interactive decision-aid smartphone smoking cessation application (app) on quit rates: a double-blind automated randomised control trial protocol. BMJ Open 2014;4:e005371. doi:10.1136/bmjopen-2014005371

- Prepublication history for this paper is available online. To view these files please visit the journal online (http://dx.doi.org/10.1136/ bmjopen-2014-005371).

Received 31 March 2014 Revised 10 June 2014 Accepted 4 July 2014

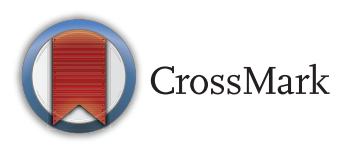

${ }^{1}$ School of Public Health, University of Sydney, Sydney, New South Wales, Australia ${ }^{2}$ Public Health and Health Informatics School, College of Health Sciences, Saudi Electronic University, Riyadh, Saudi Arabia

\section{Correspondence to} Nasser F BinDhim; nbin6641@uni.sydney.edu.au

\section{ABSTRACT}

Introduction: In a previous study exploring the feasibility of a smoking cessation application (app), we found that about $77 \%$ of the respondents from three countries were ready to quit in the next 30 days without significant differences between countries in terms of age, operating system and number of quitting attempts. However, the efficacy of smartphone apps for smoking cessation has not yet been established. This study tests the efficacy of a smartphone smoking cessation decision-aid app compared with an app that contains only smoking cessation information.

Methods and analysis: This is an automated double-blind, randomised controlled trial of a smoking cessation app that contains the eligibility requirements and baseline questionnaire and will randomise the participants into one of the two subapps (the intervention and the control). Participants will be recruited directly from the Apple app stores in Australia, Singapore, the UK and the USA. Daily smokers aged 18 and above will be randomised into one of the subapps after completing the baseline questionnaire. Abstinence rates will be measured at 10 days, 1 month, 3 months and 6 months, with the 1-month follow-up abstinence rate as the primary outcome. Logistic regression mixed models will be used to analyse the primary outcome.

Ethics and dissemination: This study was approved by the University of Sydney's Human Ethics

Committee. The results of the trial will be published in peer-reviewed journals according to the CONSORT statement.

Trial registration number: Australian New Zealand ClinicalTrial RegistryACTRN12613000833763.

\section{INTRODUCTION}

A smartphone is a mobile phone handset with advanced processing capabilities that enable it to carry out complex functions akin to those of personal computers. ${ }^{1}$ The rapid uptake of smartphones globally, their

\section{Strengths and limitations of this study}

This study provides a new method of conducting a cross-country automated randomised controlled trial with no human intervention by utilising smartphone capabilities.

- The use of an automated recruitment, randomisation and follow up in this study decreases the cost and staff burden.

- This is one of the first studies that explored the feasibility of recruiting RCT participants directly via Apple app store, and investigating a crosscountry efficacy of smartphone app for smoking cessation.

- Although we have implemented a function to prevent users from downloading the app from another device, it may not completely eliminate the possibility of contamination but hopefully will reduce it.

proximity to their users and their processing power make them potentially an optimum new delivery vehicle for health information and interventions. Smartphone ownership had reached $88 \%$ of all mobile phone handsets in Australia $^{2}$ and Singapore $^{3}$ by 2013. Ownerships also at $72 \%$ in the $\mathrm{UK}^{4}$ and $65 \%$ in the USA. ${ }^{5}$

The smartphone applications (apps) combine the advantages of computer and internet-based smoking cessation interventions but overcome many of their limitations. ${ }^{6}$ For example, users can still benefit from the computational interactive functions and static information in the apps if an internet connection is not available. ${ }^{6}$ Smoking cessation smartphone apps can host various multimedia formats, such as static and interactive-rich text, pictures, audio and video, and obtain more content when there is an internet connection without any user effort. ${ }^{6}$ 


\section{SMARTPHONE APPS AND SMOKING}

There are currently dozens of smoking cessation apps in various smartphone app stores. However, information about their quality and efficacy is limited. In an attempt to evaluate the quality of the smoking cessation apps in one app store, a study found that most of the 42 apps identified were of low quality and that none scored more than 30 out of 60 points on a smoking cessation guidelines adherence index. ${ }^{7}$ In addition, the study found that the most popular smoking cessation apps were less likely to score higher on the adherence index. ${ }^{7}$ Finally, none of the identified apps followed the guidelines to ask users for their tobacco use status, assess their willingness to quit, arrange for a follow-up and/or recommend the use of approved quitting assistance medications. ${ }^{7}$ In another study in 2012, 107 prosmoking apps were identified in the Apple app store and Google Play store. These apps show explicit images of cigarette brands and teach users how to smoke in a simulation, and some claim that they can help in quitting smoking. ${ }^{1}$ These prosmoking apps have gained popularity in 49 countries, some with strict tobacco control regulations, such as a comprehensive ban on tobacco advertisements (eg, Australia), and others with fewer tobacco control regulations (eg, Indonesia) ${ }^{8}$

\section{USE OF HEALTH-RELATED SMARTPHONE APPS AS A TOOL FOR BEHAVIOUR CHANGE AND RESEARCH}

In a recent study, we investigated the feasibility and the characteristics of those using a smartphone smoking cessation app released in the two most popular app stores (Apple App Store and Google Play), along with the app uptake over 1 year in Australia, the UK and the USA. ${ }^{6}$ We found that $77.2 \%(465 / 602)$ of the respondents were ready to quit in the next 30 days, and the majority of these had never sought professional help (eg, Quitline). ${ }^{6}$ More than half had downloaded smoking cessation apps in the past and, of that group, three-quarters had made quitting attempts (lasted at least $24 \mathrm{~h}$ ) by using an app. We also found no significant differences among countries in terms of age, operating system, number of quitting attempts and language spoken at home. ${ }^{6}$ These similarities suggest that a smoking cessation app could be effective across several countries.

Our study also confirmed the feasibility of collecting research data and recruiting participants directly from app stores. The study generated an unprompted response rate of $36.7 \%$ to our in-app questionnaire, and response rates were as high as $44.9 \%$ in Australia. ${ }^{6}$ The study suggested that adding a reminder function telling the users to complete the questionnaire or any required data collection may improve the response rate. ${ }^{6}$ Furthermore, smartphone apps have shown promising results in lifestyle-changing studies. ${ }^{9}{ }^{10}$ A weight loss pilot trial comparing a smartphone app with a website and paper diary found that trial retention was $93 \%$ at 6 months in the smartphone group, compared with 55\% in the website group and $53 \%$ in the paper diary group.
The weight change over time was significantly greater in the smartphone group at $-4.6 \mathrm{~kg}(95 \%$ CI -6.2 to $-3.0)$, compared with the diary group at $-2.9 \mathrm{~kg}(95 \%$ CI -4.7 to -1.1$)$ and the website group at $-1.3 \mathrm{~kg}(95 \%$ CI -2.7 to 0.1$).{ }^{10}$ However, it has not yet been established whether smartphone apps are also effective in helping smokers to quit.

\section{DECISION AIDS AND SMOKING}

Two studies have attempted to investigate the effect of a decision aid in helping smokers decide on quitting methods and on the quit rate. ${ }^{11}{ }^{12}$ The first study was a randomised controlled trial (RCT) ${ }^{12}$ that used a paperbased decision aid compared with no intervention. It concluded that the intervention group had more quit attempts $(\mathrm{OR}=1.52)$ and higher point prevalence abstinence after 6 months $(20.2 \%$ vs $13.6 \%$; OR=1.51). The second study ${ }^{11}$ was a non-randomised before and after study that used a computerised decision aid with $20.2 \%$ reporting that they had quit smoking after 2 months.

The aim of this Smartphone Smoking Cessation App (SSC App) trial is to evaluate the efficacy of an interactive smoking cessation decision-aid app compared with a smoking cessation static information app on quit rates.

\section{METHODS AND ANALYSIS \\ Study design}

This is an automated, double-blind, RCT to determine the efficacy of a self-help, interactive, decision-aid smartphone smoking cessation app compared with an app that contains only smoking cessation information. A single app has been developed (figure 1) that includes the baseline questionnaire and two subapps: the intervention and control apps. After completing the baseline questionnaire, eligible participants will be automatically randomised to one of the subapps.

\section{Trial design and randomisation}

Randomisation and study blinding

The app automatically randomises eligible participants (daily cigarette smokers, aged 18 years and above) to one of the two groups while keeping the two groups balanced using stratified block (age, gender, country) randomisation (figure 2). The strata will be defined by the combination of age group, country and gender. Participants and all investigators will be blinded to group allocation (double blind).

\section{Debriefing participants}

As this study includes active concealment as to group allocation and planned deception that the control group is receiving the 'best strategy' for quitting smoking, the participants who agreed to be randomised at the start of the study will be able to access both versions of this study's subapps at the end of the study. 
A

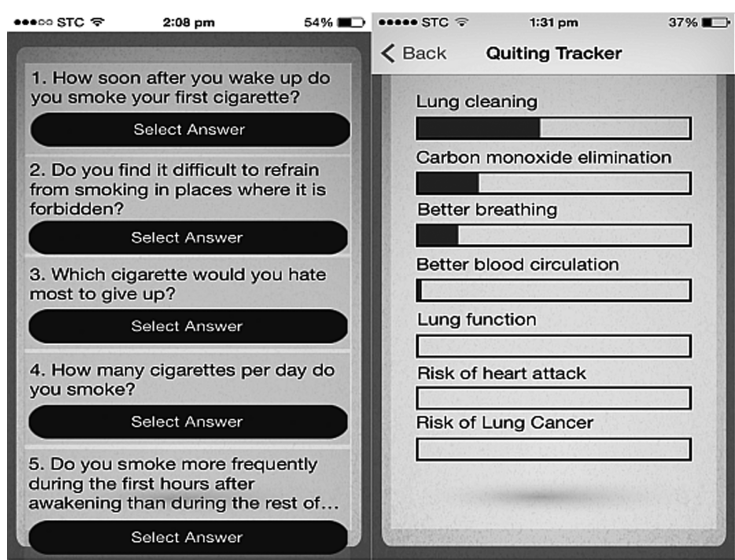

C

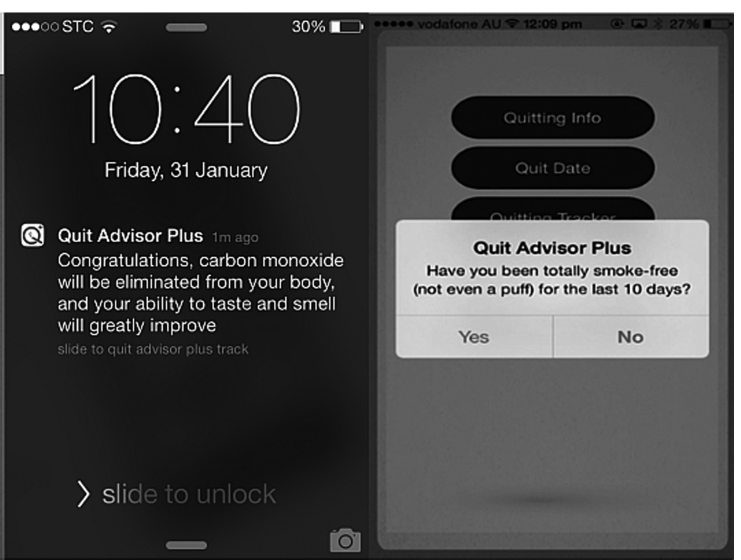

Figure 1 Screenshots of the study app ( $\mathrm{A}$ is a screenshot of the baseline questionnaire, $\mathrm{B}$ a quitting benefits tracker, $\mathrm{C}$ a notification message when a quitting benefit accomplished and $D$ the follow-up process).

\section{Study participants}

Users of the Apple App Store in Australia, Singapore, the UK and the USA will be recruited directly via the app download page in the Apple App Store. This recruitment method's feasibility was successfully tested in our previous study. ${ }^{6}$ After downloading the app, users will be asked to complete the eligibility questionnaire. If they are eligible, they can proceed to complete the remaining steps; if they are not eligible, a 'thank you' message will be displayed, and they cannot resubmit the eligibility questionnaire even if they reinstall the app.

\section{Inclusion and exclusion criteria}

The participants will be included in this study if they are daily smokers of cigarettes, 18 years old or over and from the included countries. Occasional smokers and users of other tobacco products will be excluded.

\section{Recruitment and data collection}

The app download page summarises consent information before downloading, and is also included in the terms of use agreement. The consent form and participant information sheet are included inside the app's 'About' section, so users can return to them easily at anytime. The app will include the eligibility test, baseline questionnaire, the intervention app and the control subapps.

Once the user opens the app for the first time, the app will assign the user a unique device identifier (UDI) and register the device in our secure online database. The UDI will not change if the user deletes the app or resets the device. This allows anonymous data collection, prevents duplicate enrolments and prevents contamination between groups. For example, if the user deletes the app after being randomised to one of the two groups and reinstalls the app again, the app will download the same previous data from the database to the device and the user will continue using the app in the same previous group.

As this study is fully automated, not being able to ensure that some users may download the app from another device is an unavoidable limitation. However, to monitor users who download the app onto two devices, we have implemented a server-side internet protocol that can identify the users who use different devices connected to the same internet network at similar times. This may not completely eliminate the possibility of contamination but will reduce it. To increase the response rate to the baseline questionnaire, we have implemented a reminder function that will send a notification to the user to complete the baseline questionnaire.

Although the Apple App Store limits the app downloads to specific countries, our previous study found that some users from other countries were able to download the app. We have therefore implemented a location identification function to identify the user's country the first time the user opens the app. This will be compared against the user's answer. If the user's location does not match the country answer, the user will not be included in the study. The Apple App Store will also allow for restricting the user's age for the app download, and when any user attempts to download the app, a warning message telling the user about the age restriction will be displayed and the user must agree to it before downloading the app. In addition, users will be asked about their age in the eligibility questionnaire. If the self-reported age is under the eligible age for this study, then the user will not be randomised. User answers will be sent automatically to the study's database. Participants can submit the answer to any question only once and, even if they delete and reinstall the app, their answers to previous questions are kept.

\section{The intervention}

The intervention app consists of four main features. There is both mandatory information about the pros and cons of available quitting methods and optional information about quitting benefits. Participants cannot move to the next step before confirming if they have read the provided information, and they will be asked if they have read all, some or none of the information. Second, the app will send compulsory notifications at different stages of the quitting process, 


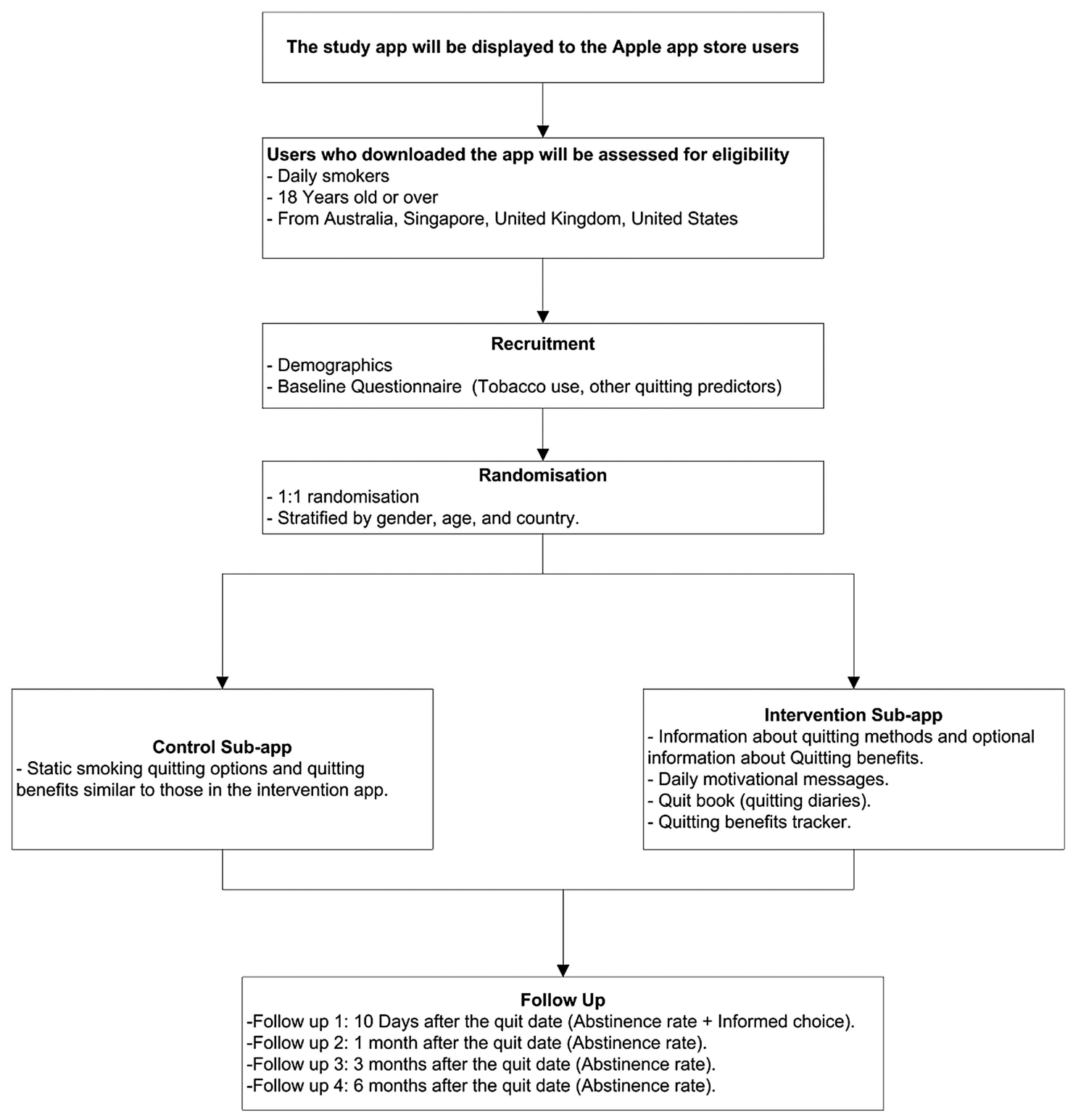

Figure 2 The trial flow chart.

for example, at the selected quitting date and daily motivational messages, using push-notification technology ${ }^{13}$ Push-notification allows the database to send messages to a specific user (similar to mobile short message service) at specified times, or after a specific task, free of cost. Third, the app includes a quit book to motivate participants to write quitting diaries (eg, craving triggers). Finally, the app includes a quitting benefits tracker that will notify the user about the health benefits gained after quitting (eg, carbon monoxide elimination after $48 \mathrm{~h}$ of quitting). Participants will also see a visual tracker for this function (figure $1 \mathrm{~B}, \mathrm{C}$ ). The app will monitor the daily usage data, including the number of times the participant opened the app, and records the number of times the participants looked at or used any function. The app will be available for the participants in the intervention group for 6 months. After this period, the app will automatically stop the data collection and participants can then use it for as long as they want.

\section{The control}

In the control subapp, only static smoking quitting options and quitting benefits similar to those in the intervention app will be provided. The app will be available for participants in the control group for 6 months. After this period, the app will automatically stop the data collection and then participants can use it for as long as they want. In addition, after the end of data collection period, participants in the control group will have access to the intervention app. The app will monitor the daily usage data, including the number of times the participant opened the app, and records the number of times the participants looked at or used any function. The control and the intervention subapps will have an 'About' section that contains the participant's information statement and consent.

\section{Primary outcomes}

1. Abstinence rate at 1 month. 


\section{Secondary outcomes}

1. Making quitting attempts (of at least $24 \mathrm{~h}$ );

2. Abstinence rate at 10 days, 1 month, 3 months and 6 months;

3. Making an informed choice on our multidimensional measure of Informed Choice for smoking at the 10-day, follow-up point;

4. Decisional conflict.

\section{Measures}

In the baseline questionnaire, sociodemographic variables will be recorded: age, sex, educational level, marital status and income level. Variables related to tobacco consumption also will be recorded: number of cigarettes smoked per day and nicotine dependence as measured by the Fageström test. ${ }^{14}$ Other variables that may affect quitting will be recorded, including depression (Patient Health Questionnaire (PHQ-2) ${ }^{15}$ and anxiety Social Phobia Inventory (mini-SPIN). ${ }^{16}$

After reviewing the quitting methods, the participants in the control and the intervention groups will be directed to answer the decisional conflict questionnaire (adapted from the SURE test) ${ }^{17}$ and then asked if they are willing to quit in the next 7 days and which of the provided options they are willing to use. Participants are then followed up on at 10 days, 1 month, 3 months and 6 months to determine how they acted on their decision. The follow-up data will be collected by pushing a notification to the participants that will be received even if the app is not running. Participants can also click on a follow-up button inside the app to initiate the follow-up process if the follow-up time has come.

The participants in both groups will be advised to complete their reading of the app within the first 3 days and to set a quit date in the next 7 days. Participants in the intervention group will be advised to use the app regularly after quitting to benefit from the self-help functions to prevent relapse. We will also record the quit date and the date of the end of the follow-up, 6 months after either the quit date or the date lost to follow-up. We will then calculate the length of the follow-up period. We will calculate the time to relapse: time interval between start date of smoking cessation and relapse or end of follow-up. Tobacco abstinence will be selfreported (not even a single puff of smoking since the quit date). ${ }^{18} 19$ If the participant relapses, then the current number of smoked cigarettes will be collected to be compared to their baseline if possible. After completing the first follow-up (at 10 days), participants in the intervention and control groups will be directed to answer the multidimensional measure of Informed Choice for smoking that measures knowledge and attitude towards smoking cessation.

\section{Sample size}

To calculate the sample size for this study, we first determined the difference between the intervention and control that we wish to detect. Based on 12 trials included in a self-help systematic review, the average abstinence rate in the control groups was $5 \%,{ }^{20}$ and the average abstinence rate in internet-assisted smoking cessation programmes was about $15 \% .^{21}$ Assuming at least a similar effect for smartphones, and as most smoking cessation trials have a small effect size, we then decided to measure at least a $10 \%$ difference between the control and the intervention groups. The proposed study requires a sample size of 672 participants to achieve $80 \%$ power at a 0.05 significance level to detect a change from $5 \%$ to $15 \%$ with a compliance adjustment of $20 \%{ }^{22}$

\section{Statistical analysis}

Analysis will be performed as an intention to treat, assuming that all participants remained in their treatment group as randomised. To handle non-responses at the follow-up, pattern-mixture models will be used as recommended by the Society for Research on Nicotine and Tobacco (SRNT), in which participants are divided into groups depending on their missing data pattern. These groups then can be used to examine the effect of the missing data pattern on the outcome(s) of interest. In addition, sensitivity analysis considering plausible models and missing data assumptions will be used to check the robustness of results across possible missing data assumptions. ${ }^{23}$ Quantitative variables will be presented by the mean and SD if they have a normal distribution, or median and range, as appropriate, and will be compared using the $\mathrm{t}$ test. Qualitative variables will be presented as percentages and CIs, and will be compared using Pearson's $\chi^{2}$ test. As this study uses an automated electronic data collection, there will be no missing values in the baseline data or other questionnaires; the app also includes a data integrity check to prevent users from entering invalid data (eg, maximum age is 99).

Regression models will be used to verify the efficacy of the intervention on the different outcome measures. Logistic regression mixed models ${ }^{23}$ will be applied for the binary outcome variables (10 days, 1 month, 3 months and 6 months point prevalence smoking abstinence), with and without adjustment for participants' baseline characteristics. Logistic regression will also be applied for the decisional conflict outcome with and without adjustment for participants' baseline characteristics.

\section{Ethical considerations}

The study will be performed in agreement with the Declaration of Helsinki. The entire study app content, participants' information statement and their consent were reviewed by the University of Sydney's Human Ethics Committee (Project No. 2013/513). As this study is fully automated, and the investigators have no direct contact with the participants, the consent will be electronically obtained. All of the obtained data are de-identified and can not be linked back to the participants. The UDI is used to connect the participants' device to the study database and will be replaced in the data set with a random ID. After completion of the study, the UDIs will be automatically deleted from the users' devices. 


\section{DISSEMINATION}

The study data collection started in May 2014. Primary and secondary analyses will start immediately after data collection is completed. The results of the trial will be published in peer-reviewed journals according to the CONSORT statement. This study has been registered in the Australian New Zealand Clinical Trials Registry (Trial ID: ACTRN12613000833763).

\section{DISCUSSION}

This study investigates the effectiveness of smartphone apps for smoking cessation, with the main outcome abstinence rate at 1-month follow-up and continuous abstinence up to 6 months, as recommended by the SRNT. ${ }^{24}$ Although the SRNT has not reached a consensus on the preferences for 6 or 12 months follow-up, they have mentioned that the major advantages of a 6-month follow-up are that several recent meta-analyses have used that length. ${ }^{24}$

In this study, we have decided to develop the app for Apple devices only, as our previous visibility study found that Apple users were more likely to download smoking cessation apps and to use them, than Android devices' users. ${ }^{6}$ In addition, the countries included in this study were also selected based on our feasibility results. ${ }^{6}$

Out of 17 internet smoking cessation RCTs included in Myung et $a l \mathrm{~s}^{21}$ meta-analyses, only one study mentioned the rationale of the achieved sample size. Although most smoking cessation studies use the same outcome measures and follow-up points, this trend is also prevalent in other smoking cessation trials. This makes it difficult for future research to calculate the sample size. Therefore, we recommend that future studies report on the rationale of the achieved sample size.

In addition to comparing the intervention's effects on quitting decision and period of quitting, this project provides a new method of conducting a cross-country automated RCT with no human intervention by utilising smartphone capabilities. The use of an automated method decreases the cost and staff burden if implemented appropriately. The study's limitation is that participants who use two devices, or who know each other, might know about the other subapp and, although measures have been placed to minimise this, we cannot eliminate this possibility.

Contributors All authors made substantial contributions to editing and revising of the manuscript. NFB was responsible for the conceptual development, the app design and development, and drafting of the manuscript. NFB and LT were responsible for the study design. NFB and KM were responsible for the statistical analysis. All authors read and approved the final manuscript.

Competing interests None.

Ethics approval University of Sydney Human Ethics.

Provenance and peer review Not commissioned; externally peer reviewed.

Open Access This is an Open Access article distributed in accordance with the Creative Commons Attribution Non Commercial (CC BY-NC 4.0) license, which permits others to distribute, remix, adapt, build upon this work non- commercially, and license their derivative works on different terms, provided the original work is properly cited and the use is non-commercial. See: http:// creativecommons.org/licenses/by-nc/4.0/

\section{REFERENCES}

1. BinDhim NF, Freeman B, Trevena L. Pro-smoking apps for smartphones: the latest vehicle for the tobacco industry? Tob Control 2014;23:e4.

2. Mackay M. Australian mobile phone lifestyle index. 2013. http://www. aimia.com.au/enews/2013/Events/AMPLI\%202013/Ampli\%202013\% 20Report_Final_October\%2024.pdf (accessed 19 Aug 2013).

3. Blackbox Research. Smartphones in Singapore: A Whitepaper Secondary Smartphones in Singapore: A Whitepaper 2013. http:// www.blackbox.com.sg/wp/wp-content/uploads/2012/05/ Blackbox-YKA-Whitepaper-Smartphones.pdf.

4. Deloitte. Secondary Exponential rise in consumers' adoption of the smartphone_can retailers keep pace? 2013. http://www.deloitte.com/ view/en_GB/uk/61d116116757f310VgnVCM1000003256f70aRCRD.htm

5. Nielsenwire. Consumer Electronics Ownership Blasts Off in 2013. Secondary Consumer Electronics Ownership Blasts Off in 20132013. http://www.nielsen.com/us/en/newswire/2013/ consumer-electronics-ownership-blasts-off-in-2013.html

6. BinDhim NF, McGeechan K, Trevena L. Who uses smoking cessation apps? A feasibility study across three countries via smartphones. JMIR mHealth uHealth 2014;2:e4.

7. Abroms LC, Padmanabhan N, Thaweethai L, et al. iPhone apps for smoking cessation: a content analysis. Am J Prev Med 2013;40:279-85.

8. BinDhim NF, Freeman B, Trevena L. Pro-smoking apps: where, how and who are most at risk. Tobacco control 2013: tobaccocontrol-2013051189

9. Brindal E, Hendrie G, Freyne J, et al. Design and pilot results of a mobile phone weight-loss application for women starting a meal replacement programme. J Telemed Telecare 2013; 19:166-74.

10. Carter MC, Burley VJ, Nykjaer C, et al. Adherence to a smartphone application for weight loss compared to website and paper diary: pilot randomized controlled trial. J Med Internet Res 2013; 15:e32.

11. Cupertino AP, Richter K, Cox LS, et al. Feasibility of a Spanish/ English computerized decision aid to facilitate smoking cessation efforts in underserved communities. J Health Care Poor Underserved 2010;21:504-17.

12. Willemsen MC, Wiebing M, Van Emst $A$, et al. Helping smokers to decide on the use of efficacious smoking cessation methods: a randomized controlled trial of a decision aid. Addiction 2006;101:441-9.

13. Apple. Local and Push Notifications. Secondary Local and Push Notifications. https://developer.apple.com/notifications/

14. Heatherton TF, Kozlowski LT, Frecker RC, et al. The Fagerström test for nicotine dependence: a revision of the Fagerstrom Tolerance Questionnaire. Br J Addict 1991;86:1119-27.

15. Kroenke K, Spitzer RL, Williams JB. The Patient Health Questionnaire-2: validity of a two-item depression screener. Med Care 2003;41:1284-92.

16. Connor KM, Kobak KA, Churchill LE, et al. Mini-SPIN: a brief screening assessment for generalized social anxiety disorder. Depress Anxiety 2001;14:137-40.

17. Parayre AF, Labrecque $M$, Rousseau M, et al. Validation of SURE, a four-item clinical checklist for detecting decisional conflict in patients. Med Decis Making 2014;34:54-62.

18. Lichtenstein E, Glasgow RE. Smoking cessation: what have we learned over the past decade? J Consult Clin Psychol 1992;60:518.

19. Fiore M. Treating tobacco use and dependence: 2008 update: Clinical practice guideline: DIANE Publishing, 2008.

20. Lancaster T, Stead LF. Self-help interventions for smoking cessation. Cochrane Database Syst Rev 2005;3:3.

21. Myung S-K, McDonnell DD, Kazinets G, et al. Effects of web-and computer-based smoking cessation programs: meta-analysis of randomized controlled trials. Arch Intern Med 2009;169:929-37.

22. Hsieh FY, Bloch DA, Larsen MD. A simple method of sample size calculation for linear and logistic regression. Stat Med 1998;17:1623-34.

23. Hall SM, Delucchi KL, Tsoh JY, et al. Statistical analysis of randomized trials in tobacco treatment: longitudinal designs with dichotomous outcome. Nicotine Tob Res 2001;3:193-202.

24. Hughes JR, Keely JP, Niaura RS, et al. Measures of abstinence in clinical trials: issues and recommendations. Nicotine Tob Res 2003;5:13-25. 